\title{
Modeling and Simulation of Dual Redundant Electro-Hydrostatic Actuation System with Special Focus on Model Architecting and Multidisciplinary Effects
}

\author{
Duansen Shangguan $^{1} \quad$ Liping Chen $^{1} \quad$ Jianwan Ding $^{1} \quad$ Yuhui Liu $^{1}$ \\ ${ }^{1}$ School of Mechanical Science and Engineering, Huazhong University of Science and Technology, China, \\ \{ahcq1990, chenlp, dingjw, yuhuiliu\} @hust.edu.cn
}

\begin{abstract}
Electro-hydrostatic actuator (EHA) is a new trend in the more electric aircraft related research works and engineering applications. As a high-performance mechatronics product, however, the physical effects of actuator behavior are multidisciplinary, coupled and strongly nonlinear. Although many commercialized multi-domain and system-level simulation packages exist, they are rarely considered and analyzed as a whole, lacking of a unified model architecture, efficient modeling forms, and comprehensive simulation verification. In this paper, Modelica is used to build a multi-domain virtual prototype of the dual redundant electro-hydrostatic actuation system (DREHAS) that consists of two EHAs in parallel, which supports multiview modeling and interdisciplinary application of the system. Finally, a simulation application case of the elevator actuation system is presented to demonstrate the effective role of Modelica models in system modeling and evaluation.
\end{abstract}

Keywords: more electric aircraft, dual redundant electro-hydrostatic actuator, working mode, system model, Modelica

\section{Introduction}

In the last decade, the power-by-wire (PBW) actuators became sufficiently mature to be applied in the more electric aircraft (Cao et al, 2012; Rosero et al, 2007). As the carrier of PBW, the EHA a hydraulic actuator driven by a dedicated pump, rather than a hydraulic network, which drives the pump to control the actuating components by adjusting the motor speed. Compared to conventional hydraulic actuators, the EHA is a typical mechatronic system with the advantages of high-power density, low load and easy modularization, which emphasizes the integration and synergy in specific domains such as mechanics, electronics, controls and hydraulics (Charles, 2017; Li, 2007). But the EHA also presents more challenges in some aspects. One of the non-negligible aspects is the physical effects of the EHA behavior are multidisciplinary, coupled and strongly nonlinear. For example, the causes of force-fighting phenomenon inherent in the DREHAS are more complicated, possibly due to voltage spikes, current transients, pressure pulses, electromagnetic interference, electromagnetic interference and mechanical losses.

Traditionally, the modeling and simulation methods for the DREHAS mainly include the theoretical modeling method based on transfer function and the cosimulation method with commercialized multi-domain simulation software. The former modeling method is to simplify and linearize some models of the system (Waheed, 2015). In this way, there is a certain gap between the processed model and the actual system, which cannot fully reflect some characteristics and working conditions of the actual system. The latter modeling method can make the model more detailed and accurate, using a variety of commercialized multidomain and system-level simulation packages. For example, the performance analysis in normal and failure modes can be achieved by co-simulation based on AMESim and Matlab (Ji et al, 2009). However, a single discipline-oriented multi-program combination often requires more effort to achieve the optimal results that a unified model system can achieve, which can lead to complexity in modeling and simulation. Moreover, the establishment of the EHA multidisciplinary unified system model is conducive to evaluating the design scheme and improving the quality of further analysis and decision making.

As is well known, Modelica implements a multidomain unified statement description of mechatronics systems, integrating energy flow, mass flow and information flow, based on the generalized Kirchhoff law (Broenink, 1999; Fritzson, 2014). The core topic of this paper is the presentation of the multi-domain unified Modelica model for the DREHA. On the one hand, it is possible to provide an appropriate method for the close cooperation of different disciplines, and on the other hand to achieve the rapid and accurate evaluation of the system characteristics, especially the phenomenon of competing forces, during the conceptual and preliminary design phases.

The rest of the paper is structure as follows. The following section covers the introduction to the structure and characteristics of the DREHAS. Section 3 shows the 


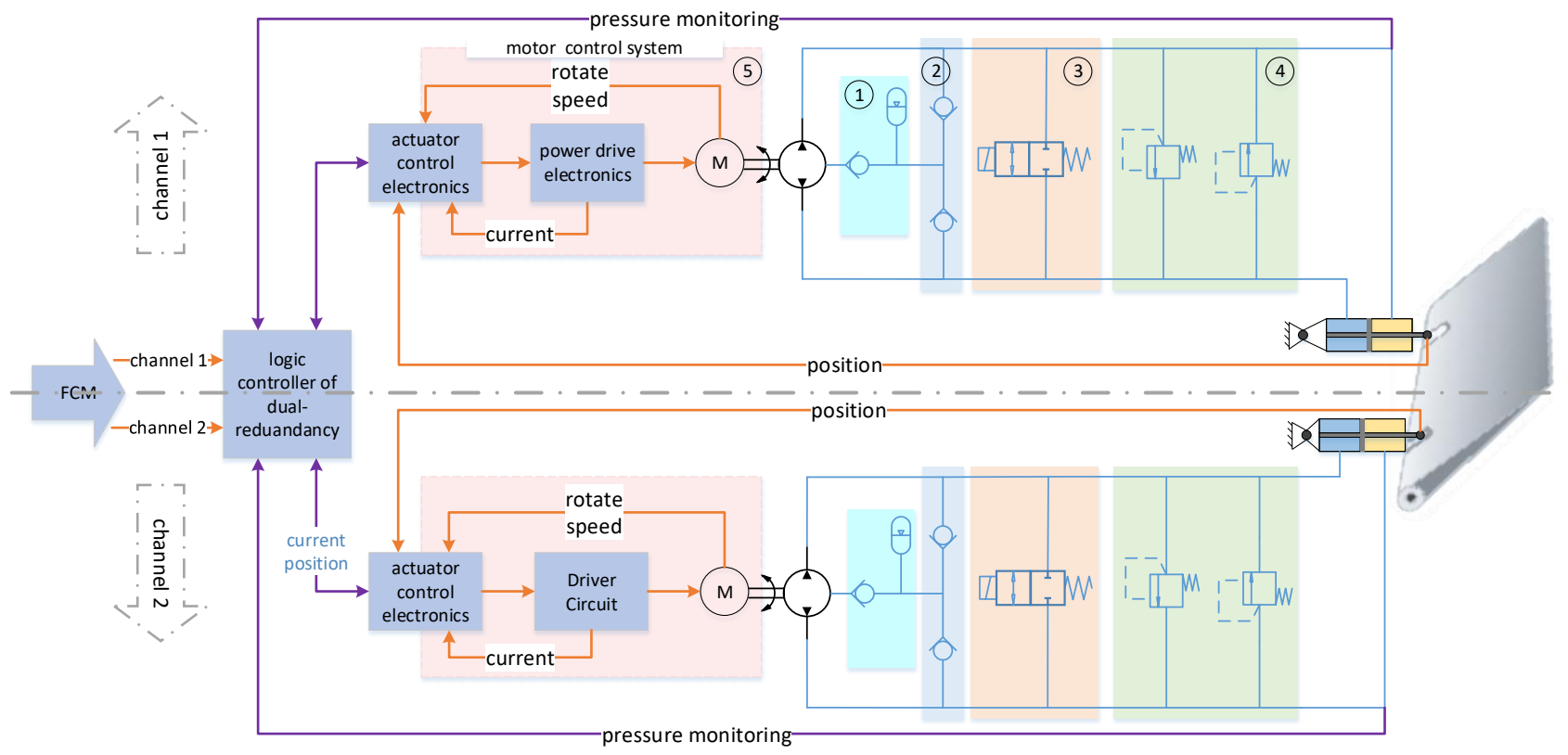

Figure 1 Schematic of a redundant electro-hydrostatic actuation system for elevator

model development process of the DREHAS. Section 4 showcases the simulation results and discussions. Finally, Section 5 gives the conclusions.

\section{The principle of the DREHAS}

The DREHAS involved in this study, as shown in Figure 1 , is composed of the two identical EHA in parallel. The controller receives the FCM instruction signals and monitors all sensor signals in real time to realize the redundancy management and control of the system. Each EHA system is a position servo control, which controls the pump output flow of the pump by adjusting the motor speed to achieve a specific actuator output displacement and speed.

In addition, the safety-critical functions such as flight control used by the DREHAS must have a very low failure rate, which requires that each channel must have fail-safe devices to allow the remaining channels to operate correctly. In the DREHAS, the safety response to faults is easily achieved with hydraulic components at low quality and low cost. Figure 1 shows the functions implemented in the DREHAS, such as oil compensation function (part (1), protection against outgassing or cavitation (part (2), oil unloading function (part (3)), bypass and safety pressure setting function (part (4)).

\subsection{Main components}

In this paper, the DREHAS for elevator actuation system consists of the following components:

- controller, which performs the closed loop control of the EHA.

- permanent magnet synchronous Motor (PMSM), which drives the quantitative plunger pump to control the pump output flow by controlling the motor speed.
- plunger pump, which converts mechanical energy into hydraulic energy that drives the actuator.

- aircraft control surface, where a change in the angle of deflection causes a change in the hinge moment on the operating surface.

\subsection{Working modes}

In general, the DREHAS has three kinds of working modes: active/active (A/A) mode (both the EHAs are actively controlled), and active/passive (A/ P) mode (one EHA is actively controlled and one passive following). In addition, the last working mode refers to the fault damped (DP) mode for the two EHA failures. When the EHA is in the damping mode, the solenoid valve will break the connection between the actuator and the pump, and the oil passage is connected at both ends of the actuator. In this case, the actuation system is equivalent to a damper.

\subsection{Control structure}

The DREHAS follows the requirements of the pilot or autopilot to drive the elevator to deflect a specific angle and overcome the uncertain interference of the external aerodynamic load. The dual redundant logic controller obtains the working state parameters of the two-channel hydraulic cylinder according to the sensor detection. In the modules of channel 1 and channel 2, the working state of the system is judged: A/A mode, A/P mode, DP mode.

The surface position setpoint and the working mode from the dual redundancy logic controller are used as inputs to the EHA. As shown in part (5) of Figure 1, when the EHA is actively controlled, PMSM adopts a linear control method involving a PID serial corrector. A commonly used controller structure consists of a 
cascade of three nested loops: a current (internal) loop, a speed (middle) loop, and a position (external) loop. In addition, current feedback, speed feedback, position feedback, and output pressure of the actuator are used for real-time monitoring and control of the EHA.

\subsection{Force-fighting phenomenon and multiple physical effects}

In the active/active mode, the two EHAs that make up the DREHAS work together to push the rudder surface. However, the magnitude of the respective output forces in the physical actual state may not be completely uniform, and the rudder surface has a large rigidity, which causes a force-fighting phenomenon between the plurality of main actuators on the same rudder surface.

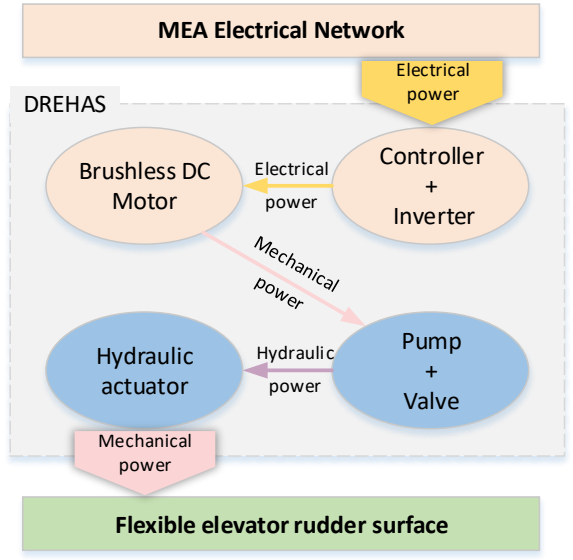

Figure 2 Energy transfer process among different physical domains in the DREHAS

In the traditional hydraulic double-residual actuation system, the force-fighting phenomenon is mainly caused by the accumulation of manufacturing and installation errors of sensors, actuators and rudder surfaces. However, the energy transfer during the operation of the DREHAS for the elevator is more complicated, as shown in Figure 2, and its control precision is more precise with 4 closed-loop controls. In this case, the factors causing force-fighting phenomenon are multidisciplinary, such as grid pollution, voltage spikes, current transients, electromagnetic interference, electromagnetic interference and mechanical losses.

\subsection{Model structures for system modeling and evaluation}

In terms of model modeling and simulation systems, choosing the right "good enough simulation model" means choosing a model with the corresponding granularity, which depends largely on the needs of the current engineering task. In many cases, a very precise system modeling is not a reasonable way to describe complex mechatronics, because even the uncertainty and cost of a relatively detailed model may be so high that its disadvantages become unaffordable compared to simpler modeling. So, the best model should be just enough to answer design questions, but not a more elaborate model.

This article focuses on issues related to system modeling and evaluation of the DREHAS, such as system function verification in different working modes, analysis of multiple physical phenomena (motor torque pulsation, current transients, pressure pulsation, rudder flutter), analysis and optimization of force-fighting phenomenon. This requires different granularity of the model of the DREHAS components. The models concerned mainly include: the motor and its controller, the plunger pump and the flexible rudder surface.

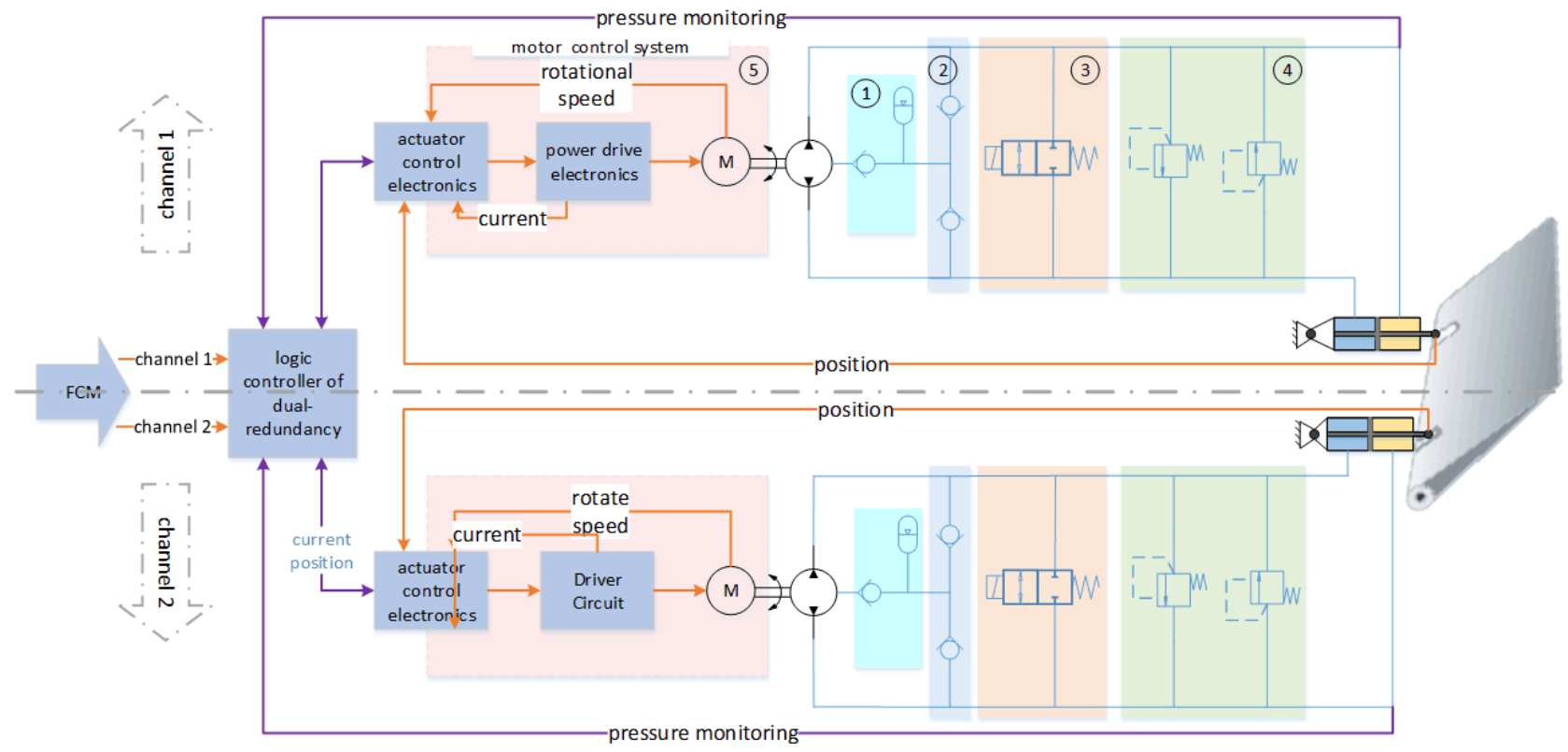

Figure 3 Realistic DREHA model in MWorks 


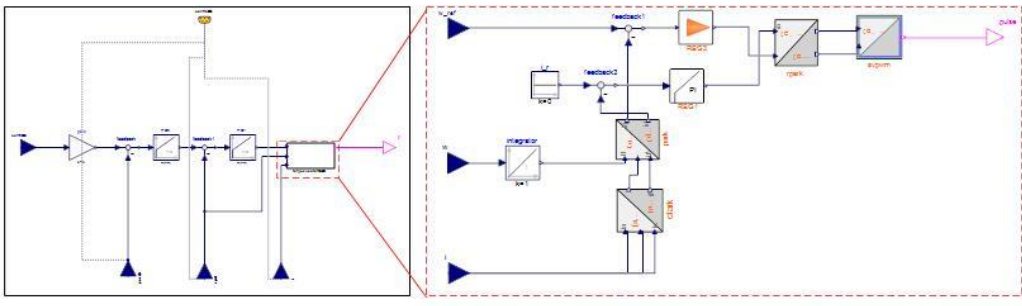

(a) controller submodel and torque vector control model

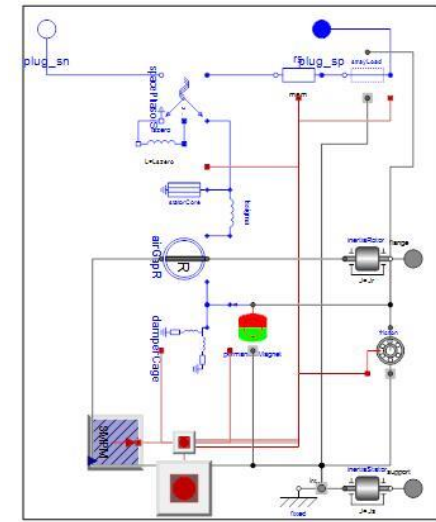

(b) permanent magnet synchronous motor(PMSM) submode

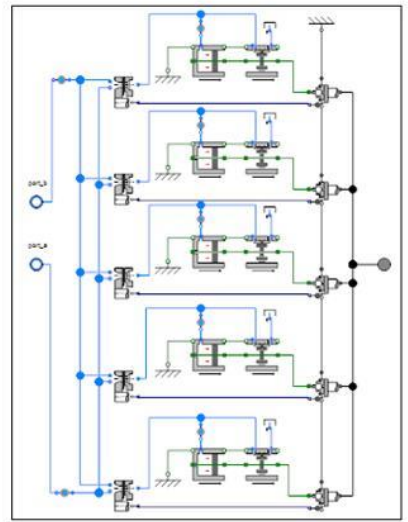

(c) plunger pump submodel

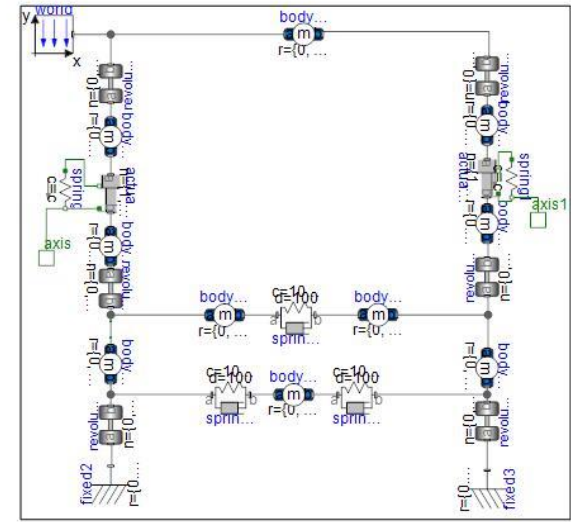

(d) flexible rudder surface model submodel

Figure 4 Five main submodels that make up the DREHAS in MWorks

\section{Model implementation and Virtual prototype}

The previous sections introduced the highly nonlinear DREHAS model architecture and multidisciplinary effects. In this section, a virtual prototype of the DREHAS for elevator control is established in the multidomain simulation environment MWorks. MWorks provides management of models and integrative solvers, where Modelica standard library version 3.2.1 and older versions can be invoked (Chen et al, 2011).

Reusing existing models can greatly improve the efficiency of modeling and enable designers to focus more on design than on detailed model development or derivation of mathematical formulas. In this paper, based on the principle structure of Figure 1, the standard library model is called as much as possible to achieve a unified model that considers multidisciplinary effects. In the light of the design requirements in the preliminary design stage, the DREHAS model, Figure 3, is divided into five main sub-models (Figure 4): the controller model, the motor model, the plunger pump model and flexible surface.

\subsection{Controller model}

The DREHAS is composed of two independently controlled the EHA. The single-channel controller model is shown in Figure 1, which uses a classic threeclosed loop control structure: the current (inner) loop, the speed (middle) loop and the position (outer) loop.

As shown in Figure 4(a), all the control loops adopt PID controller. The current loop is the innermost loop of the control law, allowing the motor current to quickly track a given current, thereby increasing system stiffness. Fast dynamic response and good tracking performance are required, but no static difference is required. The rotating speed loop is the intermediate loop of the control law, which makes the rotate speed fast track the given rotating speed, thus improving the dynamic performance of the system. The position loop is the outermost loop of the control law structure, which determines the dynamic and steady performance of the EHA, to ensure the system has fast dynamic performance and the steady state error is zero.

\subsection{Motor model}

The pump drive motor in the elevator actuation servo control system is a $270 \mathrm{~V}$ high voltage PMSM with a rated power of $10 \mathrm{KW}$ and a maximum output speed of $10000 \mathrm{r} / \mathrm{min}$. The model SM_PermanentMagnet in the Modelica standard library can be directly reused. The model considers common loss effects: heat loss of armature winding resistance, brush losses in the armature circuit, friction losses, core losses, eddy current losses, and stray load losses.

The motor driver adopts three-phase full bridge circuit to convert dc voltage into a specific PWM waveform and drive the motor. In this paper, switching dynamics of the inverter are not accounted, and an average inverter is used.

Obviously, unlike traditional hydraulic servo actuators, there are multi-domain coupling effects and high-frequency loops in the EHA, such as motor torque ripple, current transients and energy losses, all of which are considered in the Modelica model shown in Figure 4(b). 


\subsection{Plunger pump model}

Hydraulic component model can be implemented by using the Hydraulic component Design (HCD) library developed based on MWorks/Modelica. The HCD library contains 1-dimensional hydraulic components, such as pistons, spools, poppets, etc.

In this paper, the EHA uses a 5-cylinder quantitative axial plunger pump as shown in Figure 4(c). Referring to the basic structure, the plunger pump model is developed by using the HCD library and the model in the 1D Modelica.Mechanics.Translational library.

\subsection{Flexible rudder surface model}

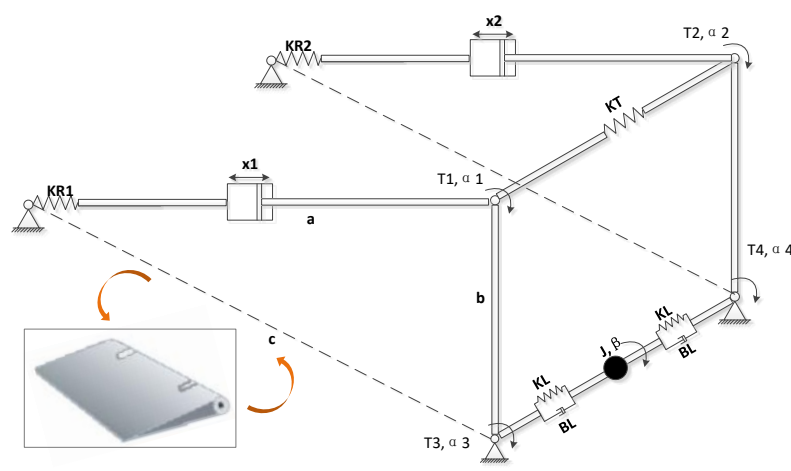

Figure 5 An elastic "structural beam" for the rudder surface

The rudder surface driven by the DREHAS cannot be considered as a simple rigid body due to the presence of two incompletely consistent driving forces. In the preliminary design stage, finite element analysis is not yet available. However, the rudder surface can be equivalent to an elastic "structural beam", as shown in Figure 5.

The relationship between the actuation displacements $x_{1}, x_{2}$ of the two parallel EHAs and the corresponding steering surface deflection angle $\beta$ is as follows:

$$
\left\{\begin{array}{l}
J \ddot{\beta}=T_{3}+T_{4} \\
T_{3}=F_{1} * \frac{b c * \cos \left(\alpha_{3}\right)}{a+x_{1}+F_{1} / K R_{1}}+T \\
T_{4}=F_{2} * \frac{b c * \cos \left(\alpha_{4}\right)}{a+x_{2}+F_{2} / K R_{2}}-T \\
T=K T\left(\alpha_{1}-\alpha_{2}\right)
\end{array}\right.
$$

Where $T_{1}$ and $T_{2}$ is the equivalent upper beam torque, $K L$ and $B L$ are the equivalent lower beam stiffness and damping, $K L_{1}$ and $K R_{2}$ are the stiffness of the two EHA fixed joints, $T_{3}$ and $T_{4}$ are the hinge moments at the rudder surface fulcrum, $\alpha_{3}$ and $\alpha_{4}$ are the deflection angles at the fulcrum of the rudder surface.

It is obvious that the numerical solution of equation (1) is highly nonlinear. Solution environment MWorks provides the equation modeling language Modelica to describe the above equations and implement automatic analytical solution of the model. Moreover, mechanical components in the standard library can be reused to build an equivalent beam model of the rudder plane, as shown in Figure 4 (d).

\section{Simulation results and discussions}

Table 1 Simulation parameters of the DREHAS model

\begin{tabular}{|l|l|}
\hline Parameters & Values \\
\hline Motor supply voltage (V) & 270 \\
\hline Maximum motor speed (r/min) & 10000 \\
\hline Swashplate inclination (deg) & 12.5 \\
\hline Armature winding resistance of motor $(\Omega)$ & 0.245 \\
\hline Armature winding inductance $(\mathrm{mH})$ & 0.008 \\
\hline Motor-pump inertia $\left(\mathrm{kg} \cdot \mathrm{m}^{2}\right)$ & 0.001 \\
\hline Displacement of pump $(\mathrm{cc} / \mathrm{rev})$ & 1.5 \\
\hline Gas pre-charge pressure (bar) & 3 \\
\hline Accumulator volume $(\mathrm{L})$ & 0.5 \\
\hline Equivalent beam stiffness $(\mathrm{N} / \mathrm{m})$ & $2.7 \mathrm{e}^{5}$ \\
\hline Equivalent beam damping $(\mathrm{Nm} /(\mathrm{rad} / \mathrm{s}))$ & 80 \\
\hline $\begin{array}{l}\text { Stiffness at the outboard fixed joints } \\
\text { (N/m) }\end{array}$ & $10 \mathrm{e}^{7}$ \\
\hline Stiffness at the inboard fixed joints $(\mathrm{N} / \mathrm{m})$ & $8 \mathrm{e}^{7}$ \\
\hline
\end{tabular}

In this section, based on the system model shown in Figure 3, the system characteristics of the DREHAS two main and fault operating modes (A/A mode, A/P mode, and DP mode) are simulated and analyzed. In addition, the force-fighting phenomenon caused by multidisciplinary effects in the A/A mode is simulated. The main parameters used in the following simulations are listed in Table 1.

Moreover, at $0.5 \mathrm{~s}$, a $0.02 \mathrm{~m}$ step signal is given to the system as the input of displacement instruction. And at $2 \mathrm{~s}$, a loading of external pneumatic disturbance of 2000 $\mathrm{N}$ is applied to the flexible rudder surface.

\subsection{Active/active mode}

In the A/A mode, the EHAs are in active control, and the two channels of the DREHAS work in parallel, driving their respective actuators to drive the rudder surface motion. The position control performance of the two EHAs are shown in Figure 6(a) indicates that the EHA almost reaches stability without overshoot at $1.5 \mathrm{~s}$, and the steady state error is less than $1 \%$. When aerodynamic disturbance occurs, the steady state of the system is broken and then stabilized again under the action of the controller (consuming 0.4s). It can be seen that the controller can quickly restore the original position and has strong anti-interference ability. In addition, the EHA is a complex mechatronic product with multidisciplinary coupling. Different from the traditional hydraulic actuator, the actuator pistons move 
more rapidly, as shown in Figure 6(b). Part of the reason is due to the mechanical inertia of the mechanical system (traditional factors); the other part is due to the high frequency response of the motor and the plunger pump (multidisciplinary factors), as shown in Figure $6(\mathrm{c}) \&(\mathrm{~d})$.

In the above simulation process, both EHA are in the ideal condition, without considering the actual multiple physical factors such as sensor error, electromagnetic interference, voltage pulsation, mechanical loss, and so on. In this case, there is no force fighting phenomenon between the two channels of DREHAS. In order to simulate the phenomenon of force fighting phenomenon, the model needs to be modified accordingly, where it is chosen to add a deviation at the motor drive current input. There is a large deviation between the two EHAs output forces, that is, there is the force fighting phenomenon (Figure 6(f)). Due to the flexibility of the

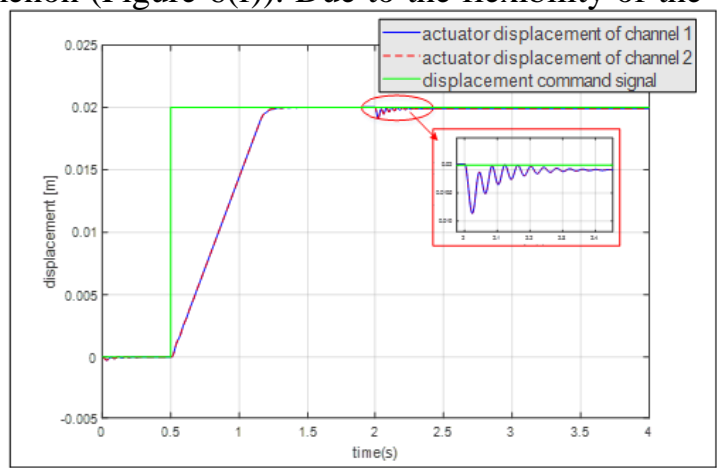

(a) Actuator displacement

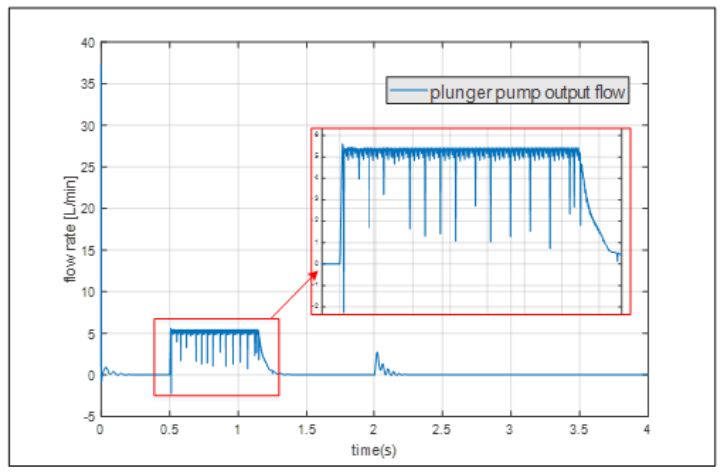

(c) plunger pump output flow

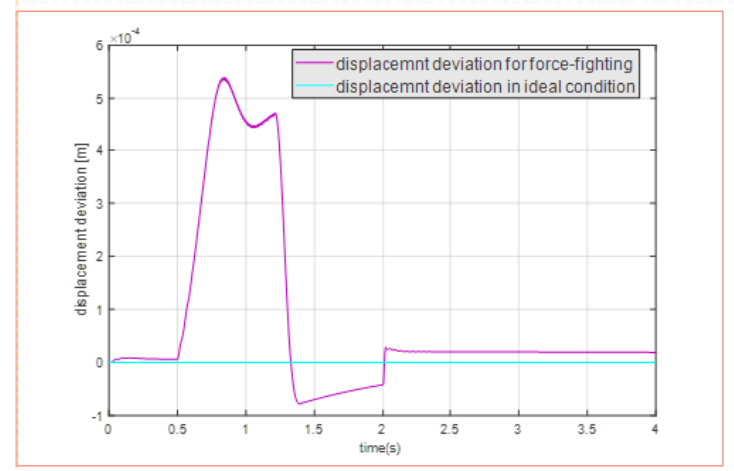

(e) displacement deviation rudder surface and the respective different driving forces, there is a certain deviation (Figure 6(e)) in the output displacement of the two EHAs.

\subsection{Active/passive mode \& fault mode}

In the A/P mode, one channel of the EHA is actively controlled and the other passively follows. For EHA in passive mode, by-pass solenoid valve connects both ends of the actuator and the chamber will be filled with fluid under the pressure of the compensator. At this time, when there is an external load on the rudder surface, the hydraulic oil of the oil return system will flow between the two cavities through the throttle valve to act as a damping. Generally, the rudder surface can respond quickly under a given load gradient. Figure 7 shows that the system is stable within $0.8 \mathrm{~s}$, so the system performance can meet the requirements.

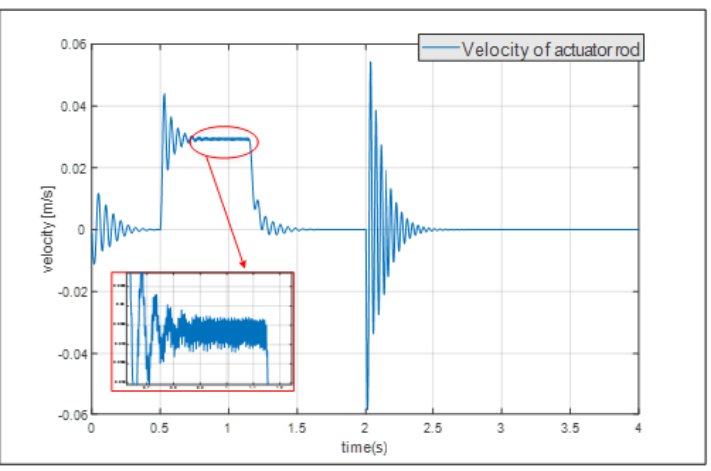

(b) Actuator rod velocity

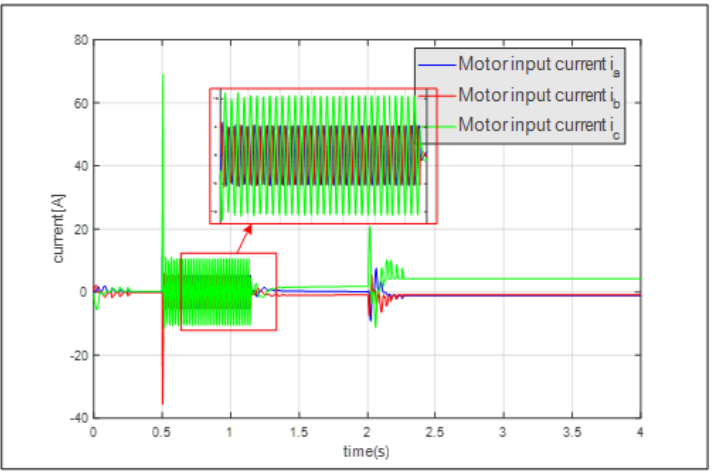

(d) PMSM drive current

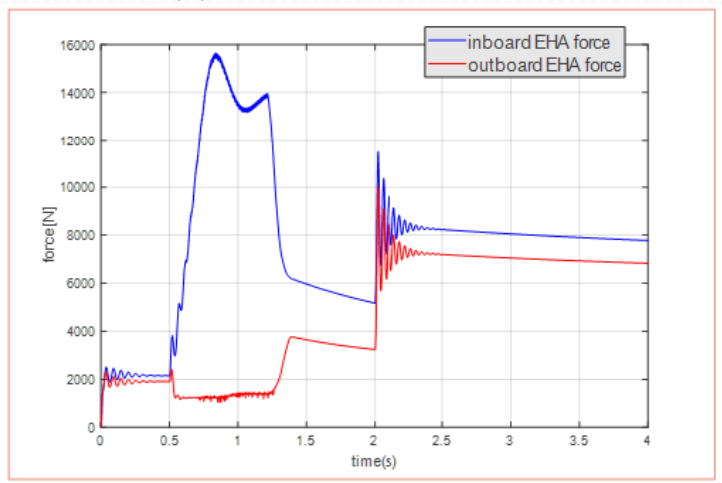

(f) fighting force

Figure 6 DREHAS response in A/A mode 


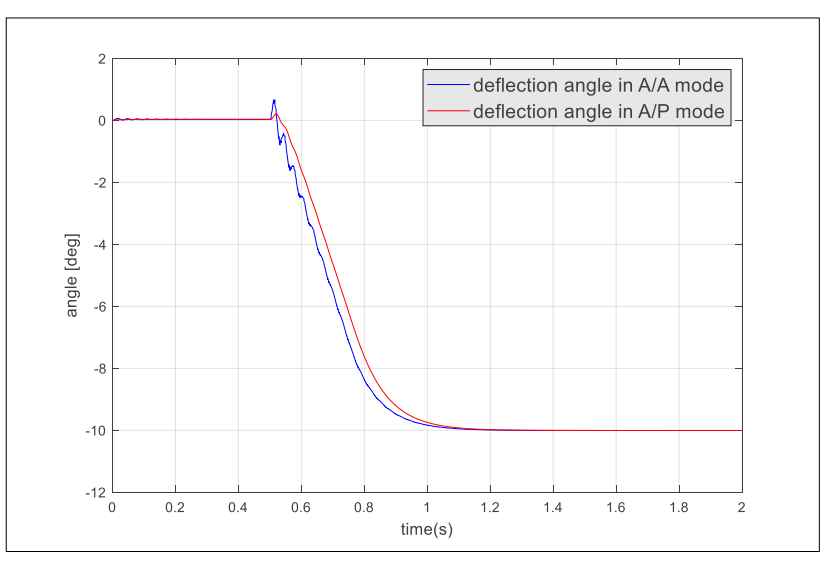

Figure 7 Deflection angle of the surface in $\mathrm{A} / \mathrm{P}$ mode

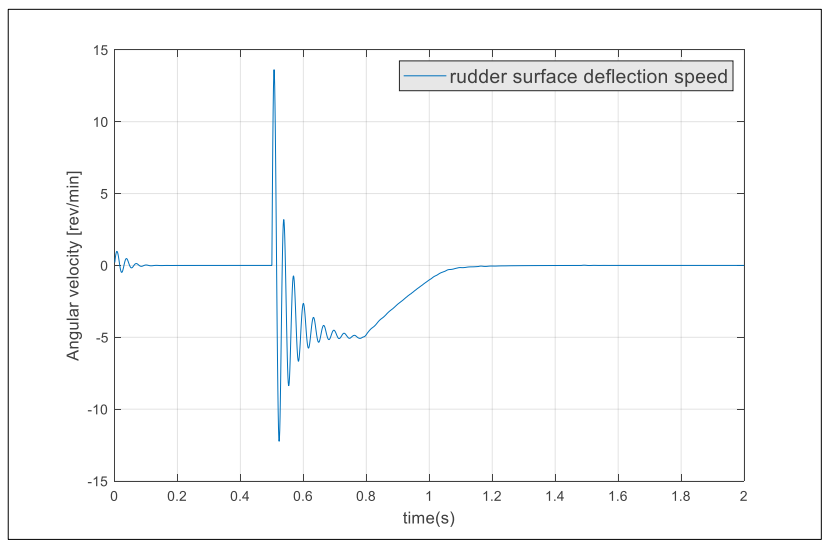

Figure 8 Angular velocity of surface deflection in the fault state

An important function of the DREHAS is to suppress the surface flutter. In normal operating mode, any EHA can use its own stiffness to suppress chatter. When the two EHAs are in a fault state, the DREHAS is in the damping mode and should be able to meet the requirements for suppressing chatter vibration through the damping circuit. Figure 8 shows the rudder surface can be stabilized rapidly under the action of external aerodynamic disturbance.

\section{Conclusions}

The DREHAS, a multidisciplinary coupled mechatronic product for more electric aircraft, is confronted with the multidisciplinary coupling problems in design. This paper presents a multi-domain unified Modelica model for multi-view modeling and interdisciplinary application in the preliminary design phase. For the multidisciplinary effects existing in DREHAS, a unified modeling language Modelica is used to establish the system model on the basis of reasonably planning the model hierarchy, defining the model interface and abstracting the model. The strong coupling and nonlinear problems are weakened to the greatest extent, which facilitates rapid simulation and verification between systems. The example of a simulation application in elevator actuation system has shown that the Modelica model not only supports the characteristic analysis of key components (motors, plunger pumps, etc.) and the overall performance evaluation of the system (system characteristics in three working modes), but also provides a suitable method for the close collaboration of experts or designers in different fields.

\section{Acknowledgements}

Thanks for the support of the members of the complex system design team in Huazhong University of Science \& Technology CAD Center.

\section{References}

Jan F. Broenink. Object-oriented modeling with bond graphs and Modelica. SIMULATION SERIES, 31: 163-168, 1999.

Liping Chen, Yan Zhao, Fanli Zhou, et al. Modeling and Simulation of Gear Pumps based on Modelica/MWorks ${ }^{\circledR}$. The International Modelica Conference, Technical Univeristy, Dresden, Germany. pp. 421-429, 2011.

Wenping Cao, Barrie Mecrow, Glynn Atkinson, et al. Overview of Electric Motor Technologies Used for More Electric Aircraft (MEA). IEEE transactions on industrial electronics, 59(9): 3523-3531, 2012. DOI: 10.1109/TIE.2011.2165453

Peter Fritzson. Principles of object-oriented modeling and simulation with Modelica 3.3: a cyber-physical approach[M]. John Wiley \& Sons, pp. 565-589, 2014.

Youzhe Ji, Song Peng, Li Geng, et al. Pressure loop control of pump and valve combined EHA based on FFIM. In Electronic Measurement \& Instruments, 2009. ICEMI'09. 9th International Conference on. IEEE, pp. 3-578, 2009. DOI: 10.1016/j.cja.2017.03.013.

Kai Li and Shaoping Wang. Multidisciplinary modeling method and simulation for Electro-Hydrostatic Actuator. Industrial Electronics and Applications (ICIEA), 2010 the 5th IEEE Conference on. IEEE, pp. 544-548, 2010. DOI: 10.1109/ICIEA.2010.5517088.

Jean-Charles Mare, Jian Fu. Review on signal-by-wire and power-by-wire actuation for more electric aircraft. Chinese Journal of Aeronautics, 30(3): 857-870, 2017.

J.A. Rosero, J.A. Ortega, E. Aldabas, et al. Moving towards a more electric aircraft. IEEE Aerospace and Electronic Systems Magazine, 22(3): 3-9, 2007. DOI: 10.1109/MAES.2007.340500

Ur Rehman Waheed, Shaoping Wang, Xingjian Wang, et al. A position synchronization control for HA/EHA system. In Fluid Power and Mechatronics (FPM), 2015 International Conference on. IEEE, pp. 473-482, 2015. 
Modeling and Simulation of Dual Redundant Electro-Hydrostatic Actuation System with Special Focus on model architecting and multidisciplinary effects 